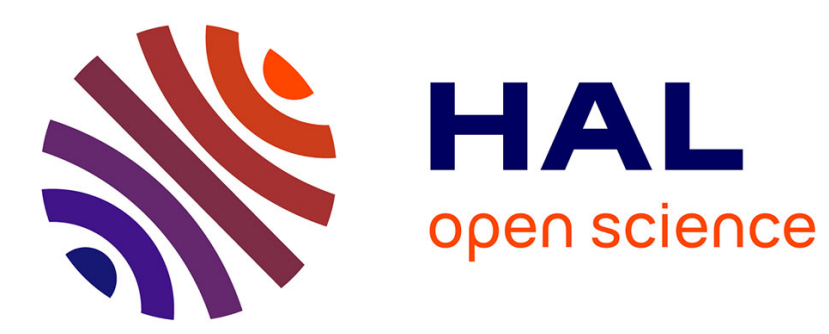

\title{
Enseignement de la géométrie à des élèves dyspraxiques : étude des conditions favorables à des apprentissages
}

Edith Petitfour

\section{To cite this version:}

Edith Petitfour. Enseignement de la géométrie à des élèves dyspraxiques: étude des conditions favorables à des apprentissages. La nouvelle revue de l'adaptation et de la scolarisation, 2017, 10.3917/nras.078.0047 . hal-02268418

\section{HAL Id: hal-02268418 \\ https://hal.science/hal-02268418}

Submitted on 20 Aug 2019

HAL is a multi-disciplinary open access archive for the deposit and dissemination of scientific research documents, whether they are published or not. The documents may come from teaching and research institutions in France or abroad, or from public or private research centers.
L'archive ouverte pluridisciplinaire HAL, est destinée au dépôt et à la diffusion de documents scientifiques de niveau recherche, publiés ou non, émanant des établissements d'enseignement et de recherche français ou étrangers, des laboratoires publics ou privés. 


\title{
Enseignement de la géométrie à des élèves dyspraxiques avec l'utilisation d'un logiciel de géométrie dynamique : étude des conditions favorables à des apprentissages
}

\author{
Edith PETITFOUR, LDAR, Université de Rouen
}

\begin{abstract}
Résumé
Les situations d'enseignement de la géométrie plane au cycle 3 font appel à différents types de tâches nécessitant la réalisation d'actions instrumentées afin de faire émerger des concepts géométriques. Les apprentissages géométriques peuvent alors sembler inaccessibles aux élèves dyspraxiques, leurs difficultés manipulatoires et organisationnelles les conduisant à l'échec dans l'exécution de leurs actions.

Nous présentons un cadre d'analyse du processus d'accès à la géométrie par la construction instrumentée avec l'utilisation d'un logiciel de géométrie dynamique. À l'aide de ce cadre et en appui sur une comparaison de constructions instrumentées dans un environnement technologique et dans l'environnement papier-crayon, nous mettons en évidence des obstacles et identifions des conditions favorables aux apprentissages géométriques pour les élèves dyspraxiques.
\end{abstract}

Mots clés :

Dyspraxie, géométrie instrumentée, apprentissage, logiciel de géométrie dynamique, enseignement

Teaching geometry to dyspraxic pupils with the use of Dynamic Geometry Software: study of conditions conductive to learning

Abstract

Teaching situations of plane geometry to fifth and sixth-grade students are based on different types of task, which require actions with instruments in order to produce geometric concepts. Learning geometry may hence appear to be inaccessible to dyspraxic pupils because their motor manipulation and organizational difficulties lead them to fail in the implementation of their actions.

In this paper, we present an analysis framework elaborated to study the process of accessing geometrical concepts through construction with instruments with the use of Dynamic Geometry Software. Thanks to this framework and thanks to a comparison of instrumented constructions in a digital environment and in a paper pencil environment, we highlight obstacles and we establish right conditions that allow dyspraxic pupils to learn geometry.

Key words:

Dyspraxia, geometry with instrument, learning, Dynamic Geometric Software, teaching 


\section{Difficultés rencontrées en géométrie par les élèves dyspraxiques scolarisés en classe ordinaire}

L'enseignement de la géométrie plane en fin d'école primaire-début de collège s'appuie sur la construction instrumentée. Les programmes scolaires du cycle 3 (MEN, 2015) précisent en effet que les activités géométriques doivent permettre aux élèves de passer d'une géométrie perceptive à une géométrie où les objets et leurs propriétés sont contrôlés par le recours à des instruments, par l'explicitation de propriétés, pour aller ensuite vers une géométrie théorique. Ainsi, les activités géométriques dont le but est la réalisation d'objets graphiques représentant des objets géométriques ont pour objectif d'enseignement l'exercice du raisonnement, à travers la mobilisation requise de connaissances géométriques.

Les situations faisant appel à différents types de tâches (reconnaître, nommer, comparer, vérifier, décrire, reproduire, représenter, construire) portant sur des objets géométriques, sont privilégiées afin de faire émerger des concepts géométriques (caractérisations et propriétés des objets, relations entre les objets) et de les enrichir (MEN, 2015, p.210).

L'utilisation d'instruments dans des activités géométriques doit conduire les élèves à mobiliser des connaissances géométriques. Cependant, cette méthode d'enseignement ne produit pas les apprentissages voulus pour les élèves dyspraxiques, alors qu'ils sont tout à fait en capacité d'entrer dans les tâches conceptuelles que requièrent les activités géométriques. Ces élèves ont, en effet, un trouble de la planification spatiale et temporelle de l'action intentionnelle et finalisée, qui se traduit par une anomalie de la réalisation gestuelle (Mazeau, 2008). Aussi, lorsqu'ils doivent utiliser des instruments, ils se montrent dans l'incapacité d'organiser leurs actions et de les réussir conformément à ce qu'ils envisagent. Ils manquent aussi d'un savoir-faire pratique concernant la prise en compte des caractéristiques des instruments manipulés de façon concrète et la prise en compte de leurs aptitudes corporelles. Malgré la répétition et l'entraînement, ils ne sont pas en capacité d'automatiser ce savoir-faire indispensable à l'obtention de la construction précise et soignée que l'on pourrait attendre.

En classe, les troubles des praxies engendrent pour l'élève des difficultés organisationnelles et manipulatoires, qui se manifestent par une grande maladresse et par une lenteur importante dans l'exécution de toutes tâches motrices nécessitant agilité, précision et rapidité. Nous donnons quelques illustrations de ces difficultés, issues de nos observations d'élèves dyspraxiques de sixième devant réaliser des constructions géométriques dans un environnement papier-crayon.

Lorsqu'il doit relier deux points à la règle, l'élève $C$ a une gestuelle saccadée et vive, sans modulation, avec parfois un freinage tardif. Sa coopération des mains est malhabile. Tout cela le conduit à des positionnements très approximatifs de l'instrument (règle mal ajustée dans l'essai 1), à des appuis mal dosés (la règle a bougé pendant le tracé dans l'essai 2 ) et à un manque de contrôle dans l'exécution du tracé (point de départ et point d'arrêt non contrôlés dans l'essai 3). À la fin de chaque essai, l'élève $C$ repère bien ce qui ne convient pas, il recommence en ajustant son action, mais il échoue autrement. II ne parvient pas à gérer simultanément les contraintes qui mèneraient immédiatement à la production souhaitée.

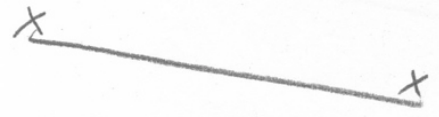

Essai 1

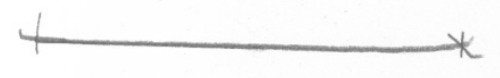

Essai 2

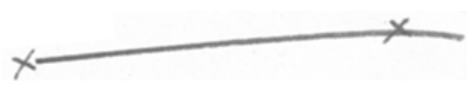

Essai 3

Lors des séances de géométrie, l'élève $M$ est la plupart du temps accaparée par des tâches scolaires périphériques au travail géométrique. Elle passe beaucoup de temps à chercher les instruments dont elle a besoin, en faisant de nombreux aller-retour de sa table encombrée d'affaires à son sac placé sous sa table. Elle rencontre souvent des problèmes de taille de crayon avec une mine qui casse sans cesse et qu'elle n'arrive pas à décoincer du taille-crayon. II n'est pas rare que les taillures se 
répandent sur son cahier et donc qu'elle ait à s'occuper de tout remettre en ordre. Elle semble dépourvue de tout bon sens pratique, par exemple lorsqu'elle tente de gommer ses tracés sans maintenir son support ou alors lorsqu'elle maintient la règle sur le trait qu'elle tente d'effacer.

L'élève $T$ ne parvient pas à réaliser de productions graphiques précises et soignées, comme on peut le voir sur la Figure 1a. Même s'il a bien utilisé les instruments attendus pour produire les propriétés géométriques voulues (équerre pour les angles droits et compas pour les reports de longueur), le dessin qu'il obtient s'écarte visuellement de la figure géométrique qu'il représente (sur la Figure 1a, il faudrait voir en $A B A^{\prime} C$ un carré). En outre, une forte dysgraphie rend pour l'élève $T$ toute communication par l'écriture calligraphique compromise. Son écrit n'est lisible ni pour l'enseignant, ni pour lui (Figure 1b).

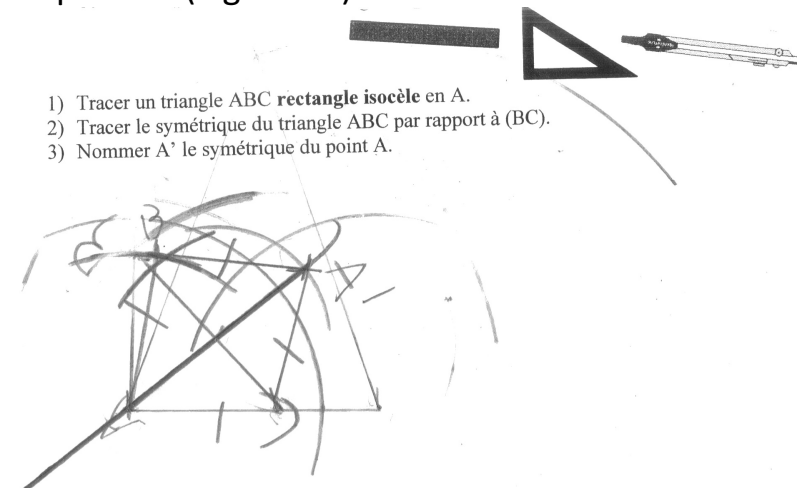

Figure 1a

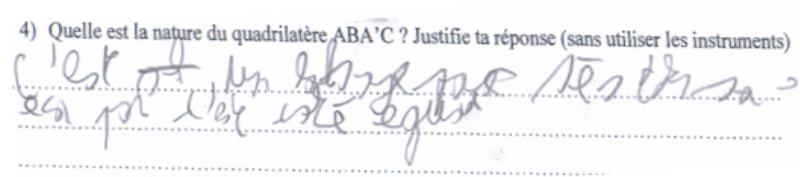

Figure $1 b$

Les élèves $C, M$ et $T$ ont tous trois des tenues d'instruments inadaptées qui rendent leurs tracés plus difficiles à effectuer. Par exemple l'équerre n'est pas positionnée de façon stable (Figure 2a), le compas est tenu par les branches (Figure $2 \mathrm{~b}$ ) ou n'est pas tenu verticalement par rapport au support (Figure 2c).

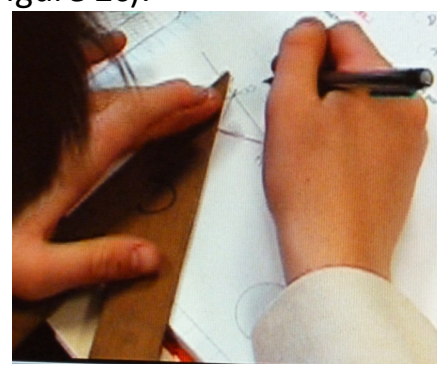

Figure 2a

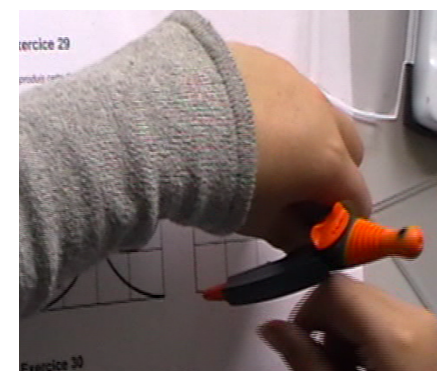

Figure $2 b$

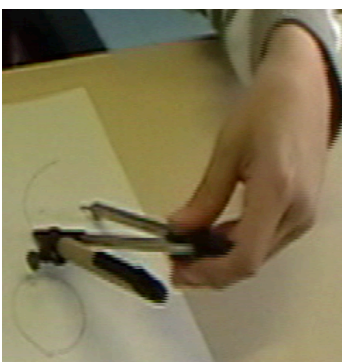

Figure 2c

Le handicap des élèves dyspraxiques compromet tout apprentissage géométrique car ils se focalisent sur les aspects manipulatoires et organisationnels des tâches de construction instrumentée, qu'ils ne parviennent pas à réaliser de façon efficace, au détriment des aspects conceptuels pour lesquels ils peuvent être performants. Ainsi que le soulignent des spécialistes en neuropsychologie :

L'enfant dyspraxique est ainsi très souvent mis en difficulté par la méthode d'enseignement, les procédures préconisées et/ou le matériel pédagogique utilisé (et non par les connaissances ou le concept à acquérir). (Mazeau \& Le Lostec, 2010, p.9)

Nous avons mis en évidence des obstacles auxquels des élèves dyspraxiques inclus en milieu ordinaire sont confrontés lorsqu'ils sont amenés à réaliser des tâches de construction géométrique dans l'environnement papier-crayon. On peut alors se demander si l'usage d'outils numériques ne pourrait pas permettre à ces élèves un meilleur accès au savoir géométrique et donc si un travail dans un environnement technologique avec l'utilisation d'un logiciel (logiciel de géométrie dynamique ou logiciel intégrant des instruments virtuels de mesure et de tracé) ne serait pas plus propice à leurs apprentissages géométriques. 


\section{Analyse de l'action instrumentée dans un environnement technologique}

Nous présentons le cadre d'analyse de l'action instrumentée que nous avons élaboré (Petitfour, 2015b) en nous appuyant sur deux approches des sciences cognitives - l'approche instrumentale en ergonomie cognitive (Rabardel, 1995) et l'approche neuropsychologique concernant le développement du geste (Jeannerod, 2011 ; Mazeau \& Pouhet, 2014). Ce cadre permet, dans des tâches de construction instrumentée, de dissocier les aspects liés à la conceptualisation en géométrie des aspects pratiques, problématiques pour les élèves dyspraxiques. II permet donc d'analyser finement les difficultés potentielles des élèves dyspraxiques dans des tâches de construction instrumentée et donc d'envisager des voies d'accès à la géométrie pour ces élèves.

\section{Cadre d'analyse de l'action instrumentée}

Une construction géométrique nécessite un enchaînement d'actions à réaliser avec des objets techniques. Les objets techniques numériques, tels des outils d'un logiciel de géométrie dynamique (exemples Figure 3a) ou des instruments de géométrie virtuels (exemples Figure 3b), apparaissent sur un écran, qui constitue l'espace perceptif de l'action. Ils sont activés grâce à la manipulation d'objets techniques matériels, tels un clavier d'ordinateur, une souris ou un pavé tactile, dans un espace d'action motrice (généralement la table sur laquelle est posé l'ordinateur).

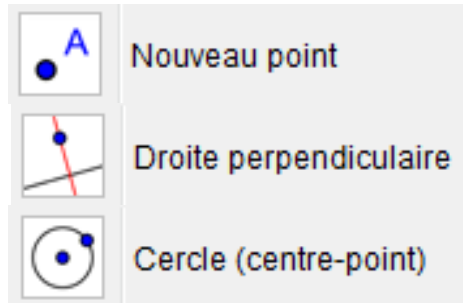

Figure 3a. Exemple d'outils du logiciel de géométrie dynamique GeoGebra ${ }^{1}$

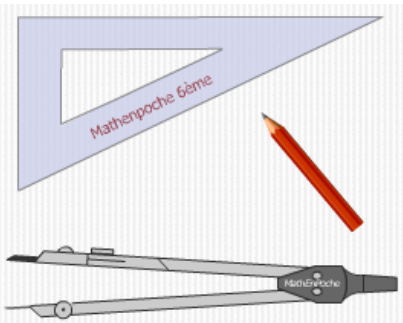

Figure $3 b$. Exemple d'instruments virtuels de MathEnPoche ${ }^{2}$

Les actions avec les objets techniques permettent de produire ou d'analyser des objets graphiques, traces virtuelles dont les caractéristiques graphiques et spatiales sur l'écran rendent compte des propriétés géométriques des objets géométriques qu'ils représentent.

Nous appelons action instrumentée l'action d'un sujet qui, dans son environnement de travail, utilise des objets techniques pour produire ou analyser un objet graphique représentant un objet géométrique. Par exemple, le tracé d'un trait droit avec l'outil "droite passant par deux points » d'un logiciel de géométrie dynamique est une action instrumentée qui permet de produire la représentation d'une droite à l'écran.

L'exécution d'actions instrumentées est le résultat de l'activation de relations entre objets géométriques, objets graphiques, objets techniques, corps du sujet et environnement. Nous considérons alors quatre composantes dans l'action instrumentée :

- la composante sémiotique (relations entre objets géométriques et objets graphiques)

- la composante technico-figurale (relations entre objets graphiques et objets techniques)

- la composante manipulatoire (relations entre le corps du sujet et les objets techniques)

- la composante organisationnelle (interactions du sujet avec des artefacts de son environnement, en lien avec l'organisation concrète de l'action instrumentée).

Nous considérons aussi la phase cognitive de l'action, antérieure à la phase de son exécution et qui comprend ses aspects préparatoires avec :

\footnotetext{
${ }^{1}$ www.geogebra.org

${ }^{2}$ http://mathenpoche.sesamath.net
} 
- l'intention d'agir du sujet (représentation du but poursuivi et du projet de l'action),

- l'intention motrice du sujet (organisation temporelle, spatiale et motrice de l'action).

Pour analyser une action instrumentée, nous considérons alors d'une part ses quatre composantes (représentées par les quatre colonnes sur la Figure 4), et d'autre part les aspects cognitifs de l'action (intention d'agir, intention motrice) ainsi que ses aspects moteurs (exécution de l'action).

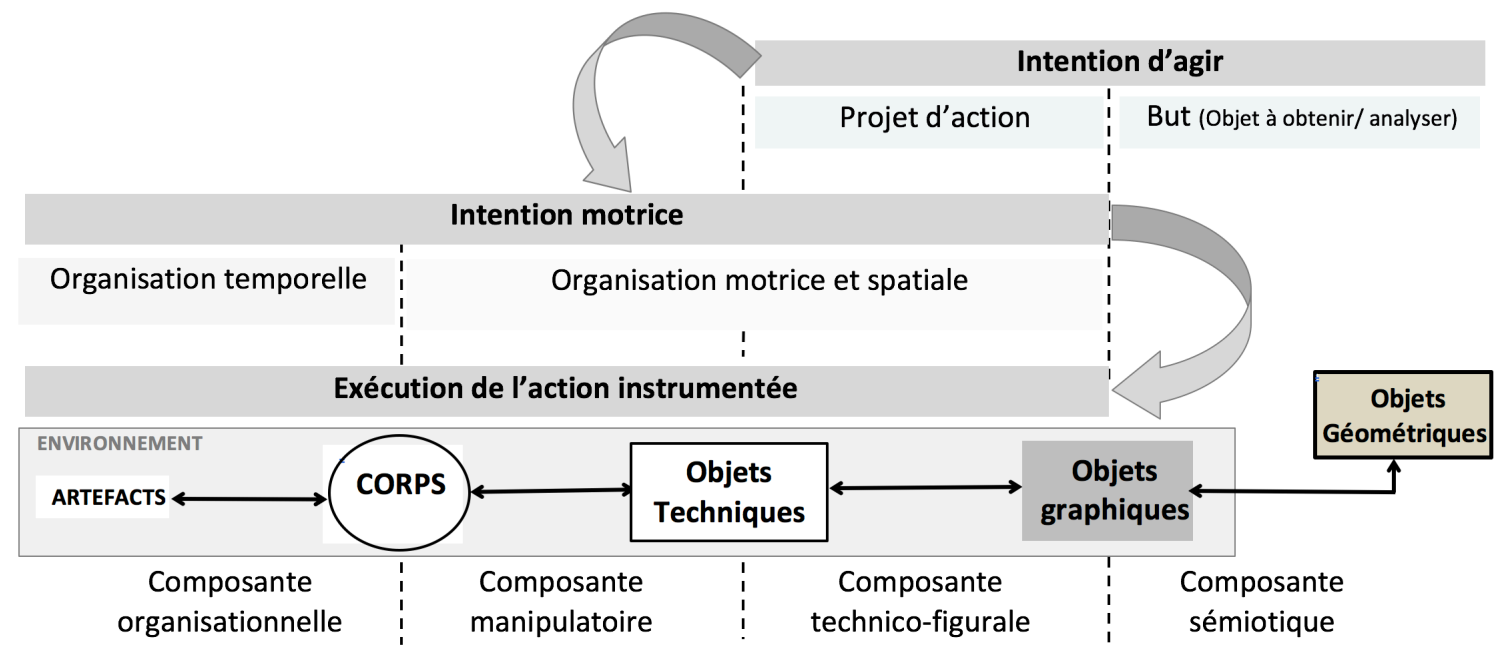

Figure 4 : Cadre d'analyse de l'action instrumentée

\section{Analyse a priori d'une action instrumentée avec un logiciel de géométrie dynamique}

Nous présentons les connaissances et compétences en jeu dans l'exécution d'une action instrumentée avec un logiciel de géométrie dynamique, à l'aide du cadre d'analyse de l'action instrumentée.

Dans son intention d'agir, au niveau de la composante sémiotique, le sujet envisage la production ou l'analyse d'un objet graphique représentant un objet géométrique. Une droite $d$ et un point $M$ étant donné, le sujet envisage par exemple le tracé de la droite $d^{\prime}$ perpendiculaire à la droite $d$ passant par le point $\mathrm{M}$ (production d'un objet graphique) ou encore il envisage de mesurer la distance entre la droite $d$ et le point $M$ (analyse de relations entre objets graphiques). L'objet à obtenir ou à analyser est le but final de l'action, il ne dépend ni de l'environnement de travail (papier - crayon ou technologique), ni des objets techniques à disposition. Le but final poursuivi engendre un projet d'actions élémentaires au niveau de la composante technico-figurale. Ce projet consiste à choisir l'objet technique et à sélectionner les objets graphiques caractéristiques. Dans les exemples précédents, on sélectionnera l'outil " droite perpendiculaire ", puis la droite d et le point M pour le tracé de la perpendiculaire et l'outil "distance ou longueur ", puis la droite $d$ et le point $M$ pour obtenir l'affichage de la distance entre la droite $d$ et le point $M$.

Dans l'intention d'agir, des connaissances géométriques sont en jeu, c'est-à-dire des connaissances relatives à la définition des objets géométriques et aux relations qui existent en eux (appartenance, perpendicularité, alignement, etc.). Dans l'exemple du tracé de la droite perpendiculaire $d^{\prime}$, il faut savoir que la relation de perpendicularité met en lien deux droites et que la droite d' est entièrement caractérisée par la donnée de la droite $d$ et du point $M$. Des connaissances graphiques, relatives à la représentation conventionnelle des objets géométriques, sont également en jeu. Ces connaissances conduisent à prélever visuellement les informations graphiques pertinentes et à les interpréter. Par exemple, il faut savoir qu'un point est représenté par une croix et que son nom est inscrit à proximité en lettre capitale. II faut savoir qu'une droite est représentée par une ligne droite et que son tracé à l'écran doit être considéré comme continu, même s'il apparaît en escalier. Enfin des connaissances techniques sont en jeu. Elles sont relatives aux fonctions de l'objet technique et à ses schèmes d'utilisation. Dans le sens donné par Vergnaud (1990), les schèmes constituent l'organisation 
invariante de la conduite du sujet pour une classe de situations, sur le plan de l'action tout comme sur celui de l'activité symbolique. Par exemple, l'outil "droite perpendiculaire " a la fonction de tracer une telle droite et pour réaliser le tracé, il faut sélectionner l'outil " droite perpendiculaire » puis la droite et le point caractérisant la perpendiculaire.

L'intention d'agir engendre une intention motrice dans laquelle l'exécution corporelle de l'action est planifiée et programmée. La programmation correspond à l'organisation motrice et spatiale de l'action et la planification à l'organisation temporelle. Au niveau de la composante manipulatoire se situent les aspects corporels de la manipulation. Par exemple, le schème de préhension est activé pour saisir la souris de l'ordinateur, avec un déplacement du bras vers l'objet cible (la souris) et une ouverture progressive de la main. Une pression (clic, double clic, appui sur une touche ou plusieurs touches du clavier) est à réaliser. Au niveau de la composante technico-figurale, doit être contrôlé le déplacement du pointeur à l'écran vers les icônes des outils du logiciel ou vers les objets graphiques. Au niveau de la composante organisationnelle se planifient les différents mouvements à réaliser, de façon successive ou simultanée.

Dans l'intention motrice, des compétences manipulatoires sont en jeu. Elles sont relatives à un savoir-faire pratique. Ce savoir-faire concerne par exemple la posture du corps par rapport à l'ordinateur ou encore la position ergonomique de la main sur la souris (Figure 5). Il concerne aussi la coordination des mouvements et des ajustements posturaux comme par exemple la coordination œil-main pour piloter le pointeur à l'écran avec le déplacement de la souris.

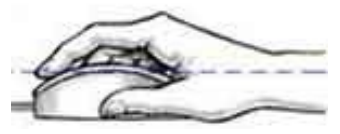

Bonne position

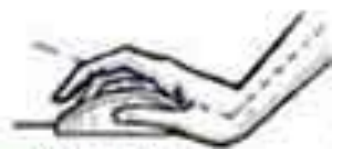

Mauvaise position

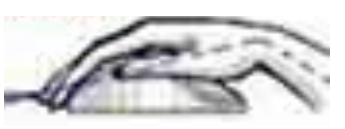

Figure 5

Enfin, des compétences visuo-spatiales sont sollicitées : les objets graphiques présents et les icônes représentant les outils du logiciel doivent être repérés par une prise d'information visuelle.

Dans la composante organisationnelle se déroule aussi la conception de l'organisation d'actions périphériques à l'action instrumentée principale. Ces actions périphériques sont nécessaires à la réalisation de l'action principale. II s'agira par exemple de brancher l'ordinateur, de l'allumer, d'ouvrir un fichier, de " déplacer le graphique ", de l'agrandir ou le réduire pour mieux visualiser la construction à l'écran, etc. Des compétences organisationnelles sont en jeu dans la réalisation de ces actions.

\section{III. Étude des obstacles et conditions favorables aux apprentissages géométriques dans un environnement technologique}

\section{Intérêts de l'usage du numérique}

Apprendre la géométrie dans un environnement technologique présente un intérêt compensatoire à des défaillances dans l'action instrumentée des élèves dyspraxiques à différents niveaux.

Au niveau manipulatoire, les aptitudes motrices requises se limitent à un nombre réduit de mouvements corporels et à une grande simplification de ces mouvements. La variété de gestes de motricité fine ainsi que la coordination bi manuelle sollicitées dans l'environnement papier-crayon sont en effet remplacées par deux types de gestes:

- des gestes de pression - relâchement de touches du clavier de l'ordinateur et des boutons de la souris, avec des appuis plus ou moins longs, réalisés avec l'index,

- de courts déplacements d'une main tenant la souris sur la table ou d'un doigt sur le pavé tactile de l'ordinateur. 
Ces seules actions motrices permettent d'activer les objets techniques numériques à l'écran, tandis que dans l'environnement papier-crayon des actions motrices beaucoup plus complexes et variées sont à mettre en œuvre pour manipuler les instruments. En outre, certaines manipulations n'ont pas lieu d'être dans l'environnement technologique, comme par exemple le maintien des instruments virtuels (règle, équerre) pendant le tracé. Les aspects manipulatoires sont donc allégés dans cet environnement. Par ailleurs, les tracés peuvent être réalisés plus rapidement que dans l'environnement papier-crayon, facilement effacés et refaits si besoin, le résultat restant soigné.

Au niveau organisationnel, la planification des tâches à réaliser à l'écran est facilitée puisque les actions à effectuer sont nécessairement successives. Pour ce qui est des instruments de géométrie virtuels, ils ont l'avantage non seulement de pouvoir être placés de façon progressive dans la position souhaitée, mais aussi de rester fixes en l'absence de commande. Par ailleurs, les objets techniques sont rangés de façon organisée et toujours à la même place : ils sont donc plus faciles à trouver une fois leur emplacement mémorisé. Cet aspect est loin d'être négligeable vu le temps important que les élèves dyspraxiques peuvent consacrer à des tâches périphériques de recherche ou d'apprêt d'instruments, au détriment d'une entrée dans l'activité géométrique.

Avec un ordinateur, l'espace perceptif que constitue l'écran est distinct de l'espace d'action motrice (la table sur laquelle se trouve le clavier de l'ordinateur et la souris), ce qui fait qu'il n'est pas obstrué par les mains du sujet lors du déplacement ou de l'activation d'un instrument, comme cela peut l'être sur le papier où les deux espaces sont confondus. De plus, la verticalité de l'écran offre un confort dans le balayage visuel et la prise de repères, notamment lorsqu'il faut passer d'une lecture du tableau de la classe à celle de l'écran. La perception visuelle peut aussi être améliorée par des agrandissements de la fenêtre de travail. Pour ce qui est du logiciel de géométrie dynamique, les objets graphiques peuvent être facilement agrandis, colorés, épaissis ou cachés, ce qui permet des tracés lisibles, et ce qui peut favoriser par exemple la focalisation du regard sur des éléments graphiques.

\section{Obstacles et conditions favorables aux apprentissages géométriques}

Au vu de l'allégement des aspects manipulatoires et organisationnels que permet l'environnement technologique, nous pouvons penser que l'usage d'outils numériques facilitera le travail des élèves dyspraxiques dans les tâches de constructions géométriques. Cela sera le cas pour ces élèves s'ils réussissent à automatiser tout le savoir-faire pratique nécessaire à la réalisation d'une construction avec le logiciel. Pour de tels élèves dyspraxiques inclus en milieu ordinaire, l'usage du numérique tels des outils d'un logiciel de géométrie dynamique ou tels des instruments de géométrie virtuels (règle, équerre, compas) manipulés avec la souris peut donc apporter un gain d'accessibilité lorsqu'il s'agit de réaliser des actions instrumentées. Cependant, les apprentissages géométriques ne pourront se réaliser que sous certaines conditions dans le cas où un élève dyspraxique travaillerait avec son ordinateur tandis que le reste de la classe travaillerait dans l'environnement papier-crayon. Une première condition est que l'élève dyspraxique et l'enseignant acceptent tous deux cette différenciation. Nous avons déjà mis en évidence plusieurs causes de réticences de la part de l'un ou de l'autre, à partir d'observations d'élèves dyspraxiques inclus en milieu ordinaire lors de séances de géométrie (Petitfour, 2015a). Ensuite, si cette différence d'environnement de travail est acceptée, il est important qu'elle soit prise en compte dans la situation d'enseignement. Nous illustrons cela par une comparaison d'une même tâche de construction réalisée dans deux environnements différents. Ainsi, prenons l'exemple de la construction d'un carré $A B C D$ à partir d'un côté $[A B]$ donné.

Nous comparons tout d'abord la première étape de la construction, à savoir la construction du côté $[A D]$ du carré, dans l'environnement papier-crayon avec comme instruments l'équerre, la règle non graduée et le compas et dans un environnement technologique avec un logiciel de géométrie dynamique avec comme outils "droite perpendiculaire ", "cercle (centre - point) ", " nouveau point " et "segment entre deux points ". Nous présentons dans le tableau suivant (Figure 6) les différentes actions élémentaires conduisant à la construction du sommet $D$ du carré. 


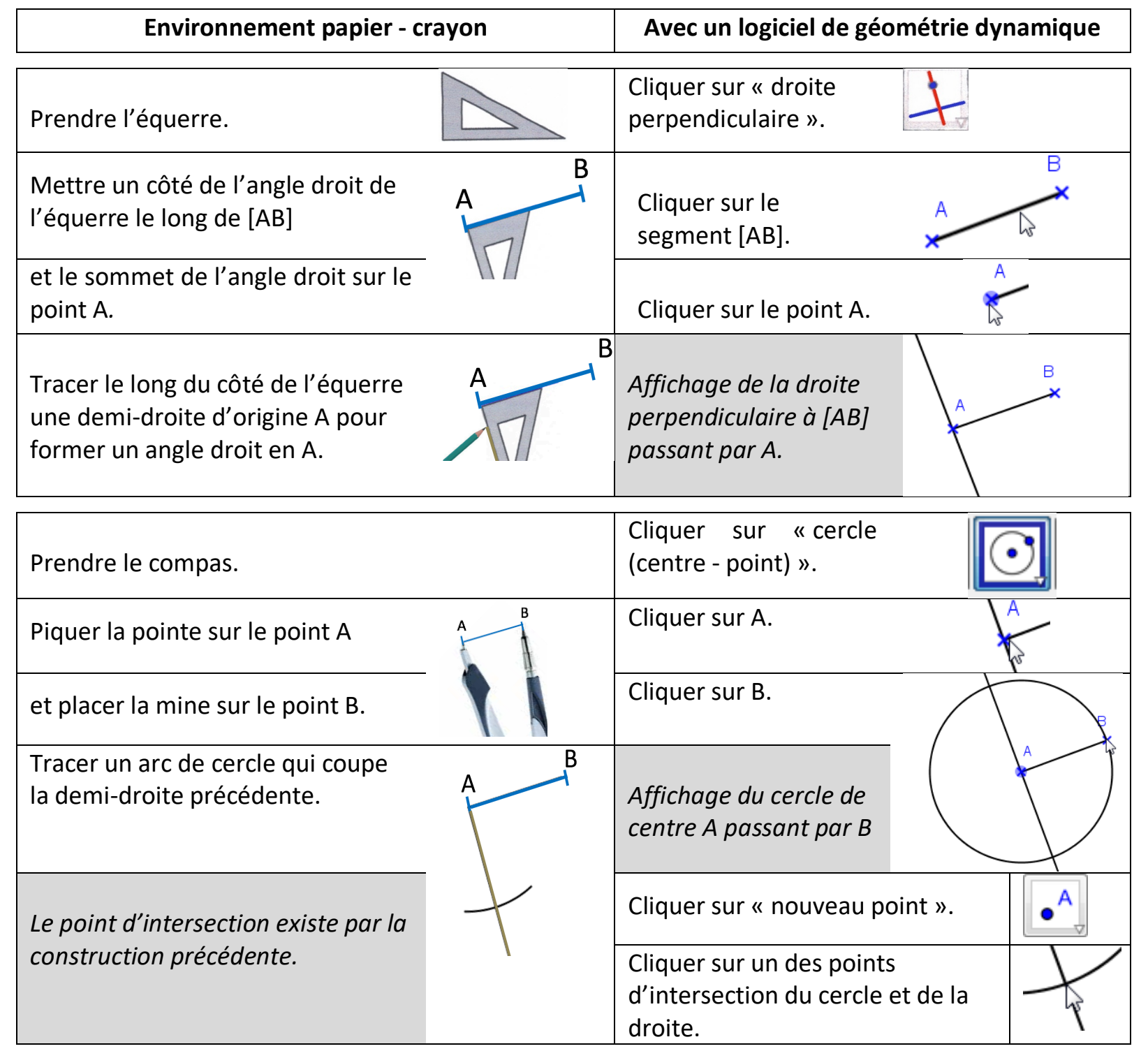

Figure 6

La première action instrumentée consiste à tracer un trait droit dans une direction perpendiculaire à la droite $(A B)$ au point $A$. Avec l'équerre, on obtiendra la représentation d'une demi-droite d'origine $A$ et avec le logiciel celle d'une droite : la trace graphique produite est donc différente dans les deux environnements. Dans l'environnement papier-crayon, il faudra anticiper sur la position du point $D$ afin d'éviter la nécessité d'un prolongement du tracé de la demi-droite d'origine $A$, cette anticipation n'est pas nécessaire avec le logiciel. Par ailleurs, une action élémentaire de tracé au crayon est nécessaire dans l'environnement papier-crayon tandis que le tracé se réalise automatiquement avec le logiciel une fois sélectionnés le segment $[A B]$ et le point $A$. L'approche conceptuelle au niveau des objets techniques utilisés pour cette première action diffère également. L'équerre en effet matérialise un angle droit tandis que l'outil " droite perpendiculaire » est représenté par une icône sur laquelle sont tracés deux traits visuellement perpendiculaires, de plus, la sélection de cet outil est accompagnée de l'affichage des termes droite perpendiculaire. Au niveau du savoir géométrique, des différences existent entre le concept d'angle droit et celui de droites perpendiculaires, tant au niveau logique que linguistique. Sur le plan logique, le concept d'angle droit est une propriété d'un objet géométrique, un angle: il s'agit d'une relation unaire, tandis que la perpendicularité est ici une 
relation ternaire entre un point (le point $A$ ) et deux droites (la droite ( $A B$ ) et sa perpendiculaire en $A$ ). Sur le plan langagier, on parlera d'angle droit avec l'équerre et de droite perpendiculaire avec le logiciel. Concernant les points communs aux deux environnements, il est nécessaire d'identifier les éléments caractéristiques de la perpendiculaire à construire : le segment $[A B]$ et le point $A$. En outre, le même type d'erreur est possible: dans l'environnement papier-crayon l'élève peut placer sa règle dans une direction contrôlée de façon visuelle pour former l'angle droit et avec le logiciel il peut utiliser l'outil " segment entre deux points " pour construire un segment ayant comme extrémité le point $A$ et qui forme perceptivement un angle droit en $A$ avec le segment $[A B]$.

La deuxième étape de la construction consiste à reporter la longueur $A B$ à partir de $A$ sur une demiperpendiculaire à la droite $(A B)$ en $A$. Dans l'environnement papier-crayon, une seule action instrumentée est nécessaire avec le tracé d'un arc de cercle de centre $A$ et de rayon $A B$ qui coupe la demi-droite précédemment tracée en le point qui sera nommé $D$. Avec le logiciel de géométrie dynamique, deux actions instrumentées sont nécessaires : la première consiste à tracer le cercle de centre $A$ passant par le point $B$ et la deuxième à créer l'un des points d'intersection du cercle et de la droite. Cette dernière action n'est pas utile dans l'environnement papier-crayon.

Au niveau graphique, les traits de construction diffèrent d'un environnement à l'autre : dans l'un, on a le tracé d'une demi-droite et celui d'un arc de cercle, dans l'autre, on a le tracé d'une droite et celui d'un cercle. Dans ce dernier cas, l'analyse visuelle des tracés peut alors très rapidement poser problème aux élèves ayant des troubles visuo-spatiaux.

Cette comparaison d'une même construction dans l'environnement papier-crayon et avec un logiciel de géométrie dynamique met en évidence des différences qu'il est important que l'enseignant prenne en compte dans son enseignement lorsqu'un de ses élèves utilise l'ordinateur comme moyen de compensation de son handicap : son discours sur la construction dans l'environnement papiercrayon n'est ni adapté ni directement transposable à la réalisation de la construction avec un logiciel de géométrie dynamique.

Un travail dans un environnement technologique avec l'utilisation d'instruments virtuels comme ceux de la Trousse Géo Tracé est visuellement plus proche d'une construction dans l'environnement papier-crayon: les instruments virtuels sont des dessins des instruments concrets et les tracés produits sont analogues dans les deux environnements. Sagot (2005) a mis en évidence l'intérêt de l'usage de ces instruments de la Trousse Géo Tracé pour donner un accès facilité à la construction géométrique d'élèves handicapés moteurs des membres supérieurs sans troubles majeurs associés. Les aspects manipulatoires sont allégés avec l'utilisation d'instruments virtuels, cependant certains usages des instruments qui seraient incorrects dans l'environnement papier-crayon peuvent conduire à des productions graphiques valides dans l'environnement technologique. Par exemple pour terminer le carré $A B C D$ (Figure 7a), l'équerre peut être glissée tout en conservant son orientation et il en est de même pour le compas. Ainsi, pour tracer la perpendiculaire à ( $A B$ ) passant par $B$, on peut avoir un positionnement de l'équerre obtenu après glissement du sommet de l'angle droit du point $A$ au point $B$ comme sur la figure $7 b$. On peut aussi obtenir le lieu du point $C$ après glissement du compas en déplaçant sa pointe de $A$ à $B$ comme sur la figure $7 c$.

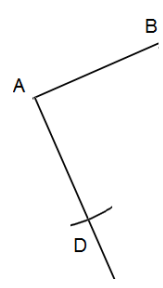

Figure 7a

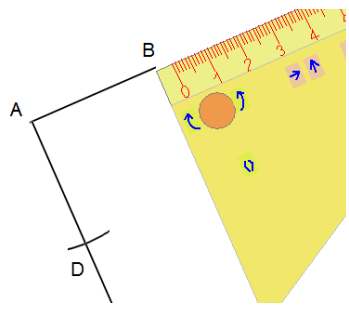

Figure $7 b$

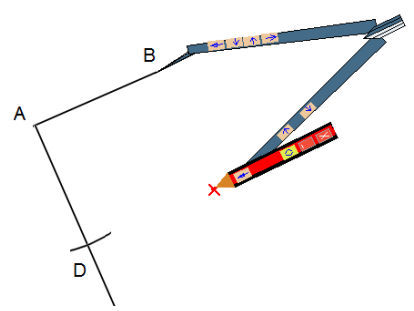

Figure 7c

\footnotetext{
${ }^{3}$ Logiciel TGT développé par Jack Sagot et Max Durand, INS HEA
} 
La conservation de l'orientation de l'équerre et du compas peut passer inaperçue pour l'élève puisqu'il ne la prend pas en charge dans le déplacement de l'instrument, ni ne la met en jeu de façon volontaire : sur la figure $7 \mathrm{~b}$, la droite $(A B)$ sur laquelle devrait être placée un côté de l'angle droit de l'équerre n'est pas représentée et sur la figure 7c, le compas permet de reporter une longueur sur une droite qui n'est pas tracée. Ainsi, de telles utilisations d'instruments conduisent à une mauvaise représentation des concepts géométriques. Cela constitue une limite de l'usage des instruments virtuels d'un logiciel pour construire des connaissances géométriques.

\section{Proposition d'un dispositif de travail en dyade}

Nous terminons notre étude des obstacles et conditions favorables aux apprentissages géométriques dans un environnement technologique par une proposition d'un dispositif de travail en dyade avec un logiciel de géométrie dynamique. Cette modalité de travail peut avoir un intérêt pour les élèves dyspraxiques qui ne parviennent pas à automatiser les aspects manipulatoires et organisationnels de I'utilisation d'un logiciel et qui, par conséquent, se focalisent sur ces aspects, tout comme ils le font dans l'environnement papier-crayon, au détriment des tâches conceptuelles qui leur sont proposées. Dans le cadre d'analyse présenté dans la partie II, remarquons que les connaissances géométriques se développent dans l'intention d'agir, de même que les connaissances graphiques et techniques en lien avec ces connaissances géométriques. Or, l'élève dyspraxique n'a pas de difficultés spécifiques à ce niveau. Aussi, nous proposons de supprimer de l'action instrumentée de l'élève dyspraxique tous les aspects relatifs à l'intention motrice en mettant en place un travail en dyade où ces aspects sont pris en charge par l'autre. Nous sacrifions ainsi délibérément le développement d'une autonomie matérielle de l'élève dyspraxique en classe pour encourager, travailler l'expression de son intention d'agir et ainsi développer son autonomie intellectuelle en lui permettant d'accéder aux concepts enseignés. L'élève aurait ainsi à :

1. activer son intention d'agir en donnant des instructions à l'autre,

2. éprouver cette action sans se préoccuper de ses caractéristiques manipulatoires fines, via l'activation de ses neurones miroirs (Rizzolatti \& Sinigaglia, 2008), en observant l'action exécutée par l'autre,

3. bénéficier d'une rétroaction de l'action réalisée par l'autre, conformément à l'intention transmise.

L'élève dyspraxique conserverait ainsi la possibilité d'élaborer les schèmes d'utilisation des instruments, pas lors d'une manipulation effective, mais lors d'une observation de cette manipulation effectuée par quelqu'un qu'il guiderait par des instructions. Cela suppose donc de développer des compétences langagières pour communiquer en mettant en jeu les apprentissages géométriques visés. Nous nous appuyons ainsi sur les compétences préservées des élèves dyspraxiques.

Dans l'enseignement, c'est l'utilisation du langage géométrique qui est visé. Ce langage, relatif aux objets, relations ou propriétés géométriques, utilise la langue géométrique constituée d'éléments d'écriture symbolique, de termes lexicaux ayant un sens spécifique en mathématiques ou de tournures syntaxiques privilégiées (Laborde, 1982). Ce langage n'est pas directement accessible aux élèves lorsqu'ils sont en phase d'apprentissage des concepts. Aussi, nous proposons d'introduire un langage proche de l'action et exprimant ce qui se passe au niveau de la composante technicofigurale : le langage technique géométrique. Ce langage est relatif à l'utilisation des outils du logiciel en lien avec des propriétés géométriques, sans mention explicite de ces propriétés ou formulation conventionnelle. Par exemple, une instruction en langage technique géométrique pour demander la construction de la droite perpendiculaire à la droite $d$ passant le point $M$ serait : sélectionne l'outil "droite perpendiculaire ", clique sur la droite d et sur le point M. Les instructions langagières peuvent être accompagnées de gestes. Nous accordons de l'importance à cette production de gestes en appui sur des travaux en psychologie qui ont mis en évidence le rôle des gestes en collaboration avec le langage dans les processus d'apprentissage (McNeill, 1992 ; Valenzeno, Alibali \& Klatzy, 
2003). Des gestes déictiques de pointage (Figure 8a) ou de parcours (Figure 8b) peuvent par exemple accompagner la désignation des objets géométriques.

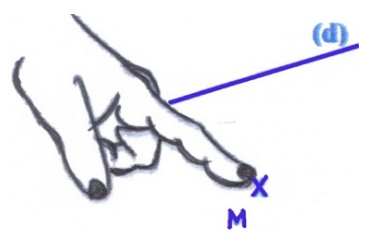

Figure 8a : pointage du point $M$

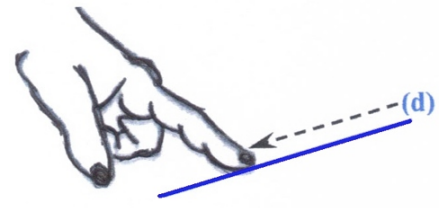

Figure 8b : parcours de la droite $d$

D’autres gestes peuvent exprimer des propriétés géométriques comme par exemple la conservation de longueurs (Figure 9a) ou la symétrie de figures (Figure 9b). Ces gestes peuvent être considérés comme des gestes pivots entre l'action et le concept géométrique (Bartolini Bussi \& Mariotti, 2008). Par exemple, les gestes représentés sur la figure $9 b$ évoquent le pliage d'une feuille de papier conduisant à superposer une figure géométrique sur une autre après retournement. Ce geste pourra être associé au concept de figures symétriques.

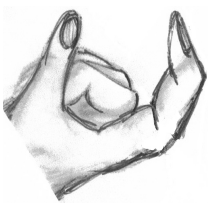

Figure 9a : conservation de longueurs

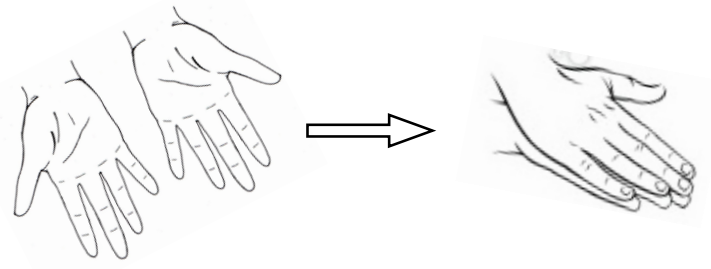

Figure $9 b$ : figures symétriques

Dans le dispositif de travail proposé, nous remplaçons pour l'élève dyspraxique la réalisation d'actions instrumentées, qui initie classiquement le travail géométrique dans les situations d'apprentissage au cycle 3, par une verbalisation accompagnée de gestes qui rendent compte de l'utilisation des instruments. Cette pratique langagière se substitue à l'action pour les élèves dyspraxiques en écartant l'activation de leurs difficultés manipulatoires et organisationnelles. Elle peut d'ailleurs aussi constituer une étape intermédiaire entre action instrumentée et formulation en langage géométrique pour des élèves ordinaires pour qui le saut entre action et validation géométrique est trop important.

\section{Conclusion}

Nous avons cherché à mieux comprendre la nature des difficultés des élèves dyspraxiques dans des tâches de construction géométrique, qu'ils soient amenés à travailler dans un environnement papiercrayon ou dans un environnement technologique. Suite à nos observations en classe d'élèves dyspraxiques de Cours Moyen et de sixième inclus en milieu ordinaire et confrontés à des activités de construction géométrique, nous pensons qu'il est contreproductif d'entraîner leurs habiletés motrices et leurs compétences organisationnelles avec l'espoir qu'ils acquièrent, à terme, des connaissances géométriques.

Nous avons étudié plus particulièrement les conditions favorables à des apprentissages géométriques avec l'usage d'un logiciel. L'utilisation d'un logiciel de géométrie dynamique peut permettre un gain d'accessibilité aux connaissances géométriques pour les élèves dyspraxiques à deux conditions. D'une part, elle ne doit pas leur poser de problèmes manipulatoires importants, d'autre part, l'exposition des connaissances faite par l'enseignant doit bien tenir compte des spécificités de cet environnement de travail dans la situation d'enseignement.

Concernant les élèves pour qui les manipulations d'instruments sont aussi coûteuses dans un environnement technologique que dans l'environnement papier-crayon, nous avons proposé un 
dispositif de travail en dyade. Ce dispositif mobilise les compétences préservées des élèves dyspraxiques, le langage et le raisonnement, et il est conçu pour que l'action ne soit plus un frein dans les situations d'apprentissage proposées à ces élèves. Nous avons expérimenté ces modalités de travail dans l'environnement papier-crayon avec deux élèves dans notre travail de thèse (Petitfour, 2015b). Les résultats sont prometteurs quant aux effets sur l'accès à des apprentissages géométriques pour les élèves dyspraxiques.

\section{Références}

BARTOLINI BUSSI M.G., MARIOTTI M.A. (2008) Semiotic mediation in the mathematics classroom : artifacts and signs after a Vygotskian perspective, in : Handbook of International Research in Mathematics Education, second revised edition, L. English, M. Bartolini Bussi, G. Jones, R. Lesh, and D. Tirosh, eds., Lawrence Erlbaum, Mahwah, NJ., pp. 746-805.

JEANNEROD M. (2011) La Fabrique des idées. Odile Jacob Sciences.

LABORDE C. (1982) Langue naturelle et écriture symbolique : deux codes en interaction dans l'enseignement mathématique. Thèse de doctorat, Université scientifique et médicale, Institut National Polytechnique de Grenoble.

MCNEILL D. (1992) Hand and Mind: What gestures reveal about thought, Chicago: Chicago University Press.

MAZEAU M. (2008) Conduite du bilan neuropsychologique chez l'enfant. Elsevier Masson.

MAZEAU M., LE LOSTEC C. (2010). L'enfant dyspraxique et les apprentissages. Coordonner les actions thérapeutiques et scolaires. Elsevier Masson.

MAZEAU M., POUHET A. (2014) Neuropsychologie et troubles des apprentissages chez l'enfant. Du développement typique aux « dys-». $2^{\text {ème }}$ édition. Elsevier Masson.

MEN (2015) Bulletin Officiel spécial n¹1 du 26 novembre 2015.

PETITFOUR E. (2015a). Enseignement de la géométrie à des élèves dyspraxiques visuospatiaux inclus en classe ordinaire. Recherches En Education, 23, 82-94.

PETITFOUR E. (2015b) Enseignement de la géométrie à des élèves en difficulté d'apprentissage : étude du

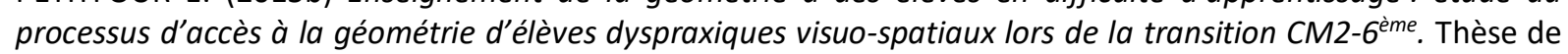
doctorat, Université Paris 7.

RABARDEL P. (1995) Les hommes et les technologies. Approche cognitive des instruments contemporains. Armand Colin.

RIZZOLATTI G., SINIGAGLIA C. (2008) Les Neurones miroirs, Odile Jacob. Poches sciences.

SAGOT J. (2005) TGT: un outil pour construire la géométrie ? Réadaptation, 122, 45-48.

VALENZENO L., ALIBALI M.W., KLATZY R. (2003) Teachers gestures facilitate students learning: A lesson in symmetry, Contemporary Educational Psychology, 28, 187-204.

VERGNAUD G. (1990) La théorie des champs conceptuels. Recherches en Didactique des Mathématiques, 10(2.3), 133-170. 